\title{
Recent COMPASS results on the nucleon longitudinal spin structure and QCD fits
}

\author{
Vincent Andrieux ${ }^{1, a}$, on behalf of the COMPASS collaboration ${ }^{\mathrm{b}}$ \\ ${ }^{1}$ CEA Saclay, IRFU/SPhN, 91191 Gif-sur-Yvette, France
}

\begin{abstract}
The latest measurements of the proton longitudinal spin structure function, $g_{1}^{p}$, in the deep inelastic (DIS) regime are presented. They improve the statistical accuracy of the existing data and extend the kinematic domain to a lower value of $x$ and higher values of $Q^{2}$. A global NLO QCD fit of all $g_{1}$ world data on the proton, deuteron and neutron has been achieved. The results give a quantification of the quark spin contribution to the nucleon spin, $0.26<\Delta \Sigma<0.34$ at $3(\mathrm{GeV} / c)^{2}$ in $\overline{\mathrm{MS}}$ scheme. The errors are dominated by the uncertainty on the shape of the functional forms assumed in the fit. A new verification of the fundamental Bjorken sum rule is obtained at a $9 \%$ level, using only COMPASS $g_{1}$ proton and deuteron measurements. Preliminary results of a reevaluation of the gluon polarization $\Delta g / g$ are presented. The analysis is based on double spin asymmetry of high- $p_{T}$ hadron production cross-sections in the DIS regime. A positive value of $\langle\Delta g / g\rangle=0.113 \pm 0.038 \pm 0.035$ is obtained at leading order at $x \sim 0.1$. In parallel, the double spin asymmetry in the photoproduction regime is also studied. Finally, preliminary results on quark fragmentation functions into pions extracted from a $\mathrm{LO}$ fit of pion multiplicities in semi-inclusive DIS are presented.
\end{abstract}

\section{Introduction}

The nucleon is a dynamic object composed of quarks and gluons. Its spin $1 / 2$ can be decomposed as follows: $1 / 2=1 / 2 \Delta \Sigma+\Delta G+L_{z}$ where $\Delta \Sigma=\Delta(u+\bar{u})+\Delta(d+\bar{d})+\Delta(s+\bar{s})$ represents the contribution from the quark polarisation, $\Delta G$ the one from the gluon spin and $L_{z}$ the contribution from the orbital angular momenta of the quarks and the gluons. We present here measurements related to $\Delta \Sigma$ and $\Delta G$, as well as results of a global QCD analysis focused on the same quantities.

\section{Spin dependent structure function $g_{1}^{p}$}

Four structure functions are used to parametrise the lepton-nucleon deep inelastic scattering $\left(l N \rightarrow l^{\prime} X\right)$ cross-section. Using longitudinally polarised lepton beam and nucleon target, one can measure a double spin asymmetry of cross-sections and extract the longitudinal spin structure function of the nucleon, $g_{1}$. The data were obtained using the $160 / 200 \mathrm{GeV}$ CERN muon beam, the long solid polarised deuteron $\left({ }^{6} \mathrm{LiD}\right)$ and proton $\left(\mathrm{NH}_{3}\right)$ targets, and the COMPASS spectrometer [1].

\footnotetext{
a e-mail: vincent.andrieux@ cern.ch

be-mail: fabienne.kunne@cern.ch,andrea.bressan@cern.ch
} 
All COMPASS results are shown in Fig. 1. The left plot shows deuteron results [2] (in red) compared to the SMC ones. The right plot shows proton data: the preliminary results from COMPASS 2011 data (in red) taken at $200 \mathrm{GeV}$ [3], the 2007 ones [4] (in blue) taken at $160 \mathrm{GeV}$, and the older SMC ones taken at $190 \mathrm{GeV}$. The $200 \mathrm{GeV}$ results extend the kinematic domain towards a smaller value of the Bjorken scaling variable $x$ (down to 0.0036). They also improve the statistical precision of the previous COMPASS measurements by about a factor 2 .
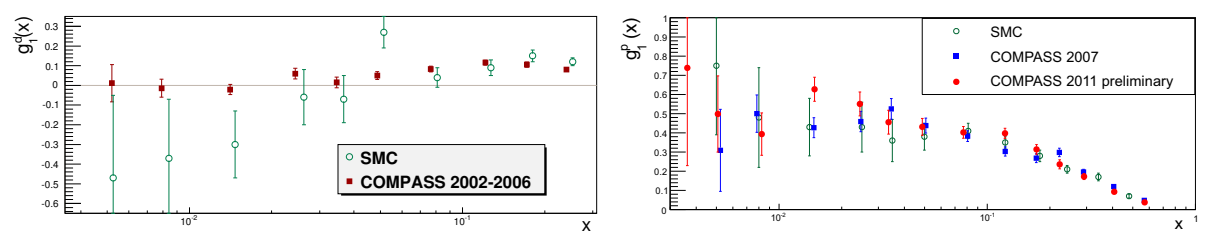

Figure 1. Comparison at the measured $Q^{2}$ of the deuteron (left) and proton (right) spin-dependent structure functions as a function of $x$ for COMPASS data (closed symbols) and SMC data (open symbols).

\section{Bjorken sum rule}

Combining the COMPASS proton and deuteron results, one can perform an evaluation of the Bjorken sum rule. It is a fundamental QCD prediction relating the first moment of the proton and the neutron spin structure functions

$$
g_{1}^{N S}=2\left(g_{1}^{p}-\frac{g_{1}^{d}}{1-1.5 \omega_{D}}\right)
$$

to the ratio of the axial-vector to vector weak coupling constants under $\mathrm{SU}(2)_{\text {flavour }}$ symmetry:

$$
\Gamma_{1}^{N S}=\int_{0}^{1} g_{1}^{N S}\left(x, Q^{2}\right) d x=\frac{1}{6} C_{1}^{N S}\left(Q^{2}\right)\left|\frac{g_{A}}{g_{V}}\right|
$$

with $g_{1}^{N S}$ the non-singlet spin structure function and $C_{1}^{N S}$ a Wilson coefficient calculable up to the third order in $\alpha_{s}\left(Q^{2}\right)$ in perturbative QCD. This sum rule has three interesting features: it is decorrelated from $\Delta g$ as it is a non-singlet quantity, it is less affected by experimental systematic uncertainties as it is the difference between two measurements and it tests the well established $\mathrm{SU}(2)_{\text {flavour }}$ symmetry. To perform the evaluation of $\Gamma_{1}^{N S}$, the $g_{1}^{N S}$ data points are included in a NLO QCD fit to get them evolved to a common scale $\left\langle Q^{2}\right\rangle=3(\mathrm{GeV} / c)^{2}$ and to complete the value of the integral outside the measured range (Fig. 2). The preliminary first moment is found to be $\Gamma_{1}^{N S}=0.181 \pm 0.008 \pm 0.014$, where $94 \%$ is coming from the measured range. It corresponds to a value of $g_{A} / g_{V}=1.220 \pm$ 0.053 (stat.) \pm 0.095 (syst.). Compared to the value from neutron $\beta$-decay measurements $(1.2701 \pm$ 0.0025) [5], the Bjorken sum rule is validated with $9 \%$ of accuracy.

The dominant uncertainty comes from the experimental systematic uncertainties: beam and target polarisation and target dilution factor (amount of polarisable material in the target).

\section{Global NLO QCD fits of $g_{1}$ world data}

The new $g_{1}^{p}$ and $g_{1}^{d}$ measurements are shown in Fig. 3 as a function of $Q^{2}$ for the various values of $x$. The COMPASS contribution is very significant in the low $x$ and large $Q^{2}$ regions, barely explored 

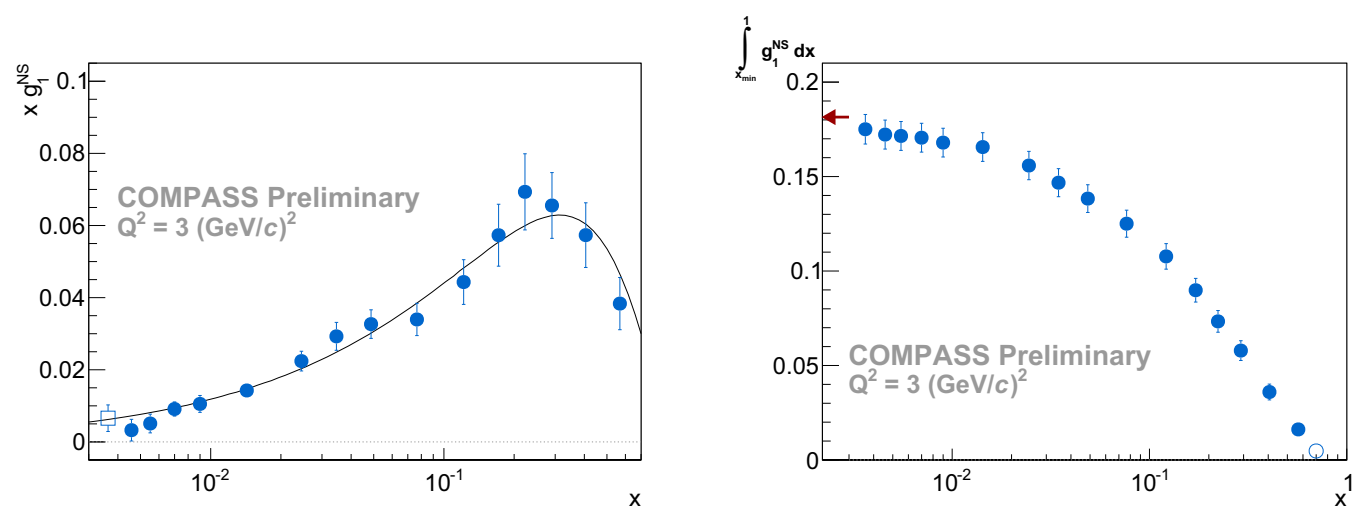

Figure 2. Left, $g_{1}^{N S}$ data points evolved at $\left\langle Q^{2}\right\rangle=3(\mathrm{GeV} / c)^{2}$ with the result of the NLO QCD fit [6]. Right, $\Gamma_{1}^{N S}$ as a function of the lower bound of the integral.
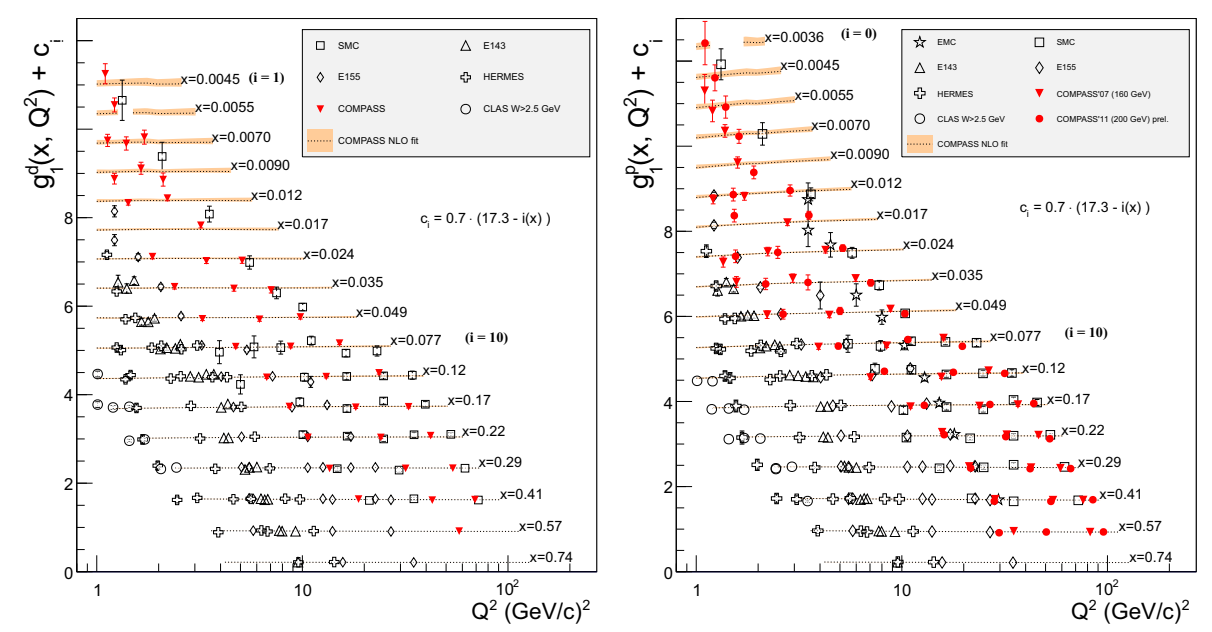

Figure 3. $g_{1}^{d}$ (left) and $g_{1}^{p}$ (right) world data as a function of $Q^{2}$ for various $x$ values. The dotted lines represent the solutions of a COMPASS global NLO QCD fit (see text).

by other experiments. The $200 \mathrm{GeV}$ data particularly complete the $Q^{2}$ range already accessed by the SMC experiment and slightly extend it up to $\sim 100(\mathrm{GeV} / c)^{2}$. These world proton and deuteron $g_{1}$ measurements are included together with the $g_{1}^{n}$ results and the COMPASS open charm $\Delta g / g$ NLO extraction [7] in a NLO global QCD fit in order to extract the helicity distributions of quarks and gluons. A functional form is assumed at an initial scale $Q_{0}^{2}$ for the singlet, the triplet and the octet SU(3) flavour combinations as well as for the gluon distribution. The first moments of the nonsinglet distributions are fixed to the values of the axial-vector weak couplings measured in neutron and hyperon $\beta$-decay. A positivity condition is also applied to $\Delta g$ and $\Delta(s+\bar{s})$ distribution to constrain the functional forms in the high- $x$ unmeasured region. The evolution is made in the $x$ and $Q^{2}$ space in the $\overline{\mathrm{MS}}$ factorisation scheme (program \#1 taken from [8]). Depending on the choice of the functional 
forms at the initial scale, three classes of equally likely solutions are found. They correspond to a positive, compatible with zero and negative $\Delta G$ value, that ranges from -1 to 0.5 at $Q^{2}=3(\mathrm{GeV} / c)^{2}$. Consequently, the value of $\Delta \Sigma$, correlated to $\Delta G$, ranges between 0.26 and 0.34 at the same scale. The dominant uncertainty in this extraction is coming from the choice of the functional forms postulated at the initial scale as they are barely discriminated by the accuracy of the present inclusive data.
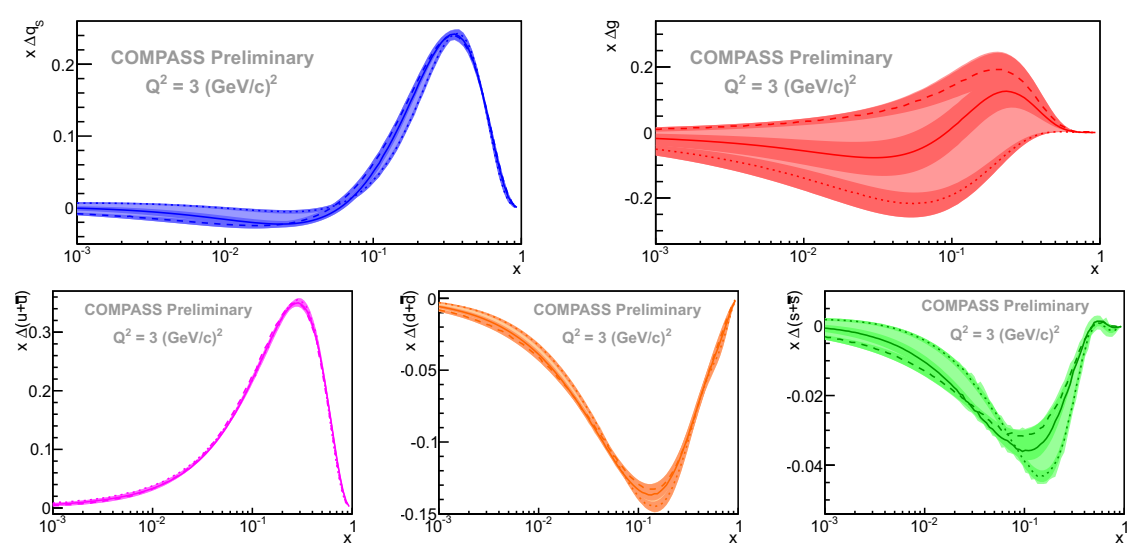

Figure 4. Top: Helicity distributions of the quarks (left) and the gluons (right) at $3(\mathrm{GeV} / c)^{2}$ in $\overline{\mathrm{MS}}$ scheme. Bottom: Helicity distribution per flavour at the same scale and scheme for $\Delta(u+\bar{u}), \Delta(d+\bar{d}) \Delta(s+\bar{s})$. Dashed (solid, dotted) lines correspond to positive (compatible with zero, negative) $\Delta G$ solutions. Dark and light colour bands correspond to the propagation of the statistical uncertainty of the data and to the combined statistical and systematic uncertainties (mainly due to the choice of the functional forms).

\section{Measurements of the gluon polarisation $\Delta g / g$}

\subsection{Measurements in the DIS regime via $A_{L L}(x)$}

Hadron production cross-section in the deep inelastic scattering regime is dominated by three subprocesses at LO: the leading process LP (photoabsoption by a quark), the QCD Compton QCDC (gluon radiation by a quark) and the photon-gluon fusion PGF (quark-antiquark pair creation). The corresponding spin asymmetry reads:

$$
A_{L L}\left(x_{B j}\right)=R_{P G F} a_{L L}^{P G F} \frac{\Delta g}{g}\left(x_{g}\right)+R_{L P} D A_{1}^{L O}\left(x_{B j}\right)+R_{Q C D C} a_{L L}^{Q C D C} A_{1}^{L O}\left(x_{c}\right)
$$

where $R_{i}$ are the fraction of sub-processes, $a_{L L}^{i}$ the spin asymmetry of the partonic cross-sections and $\mathrm{D}$ the depolarisation factor. The variables $x_{B j}, x_{C}$ and $x_{g}$ represent the fraction of nucleon momentum carried by the interacting particle for the LP, QCDC and PGF processes, respectively. All values of $p_{T}$ of the leading hadrons are considered in this analysis to supply clean samples of LP (low $\left.p_{T}\right)$ and PGF (high $p_{T}$ ). A neural network approach trained on Monte-Carlo (LEPTO+FLUKA) data is used to extract simultaneously the signal (PGF) and the backgrounds (LP and QCDC) [9]. The preliminary result obtained at LO gives $\Delta g / g=0.113 \pm\left. 0.038\right|_{\text {stat. }} \pm\left. 0.035\right|_{\text {syst. }}$ (Prel.), at the average scale of the data, $\left\langle Q^{2}\right\rangle=3(\mathrm{GeV} / c)^{2}$ and at the average gluon momentum fraction, $x_{g} \simeq 0.01$. This result is at almost three statistical standard deviations from zero, in agreement with the latest results of NLO 
QCD fits including $p p$ RHIC spin data $[10,11]$ in the same $x_{g}$ range. The data are split in three $x$ bins. They are shown in Fig. 5 left (in red) together with other direct extractions of $\Delta g / g$ at LO from other measurements. In Fig. 5 right, the same data (in red) are compared to the latest COMPASS NLO global QCD fit (which do not include this result) described in section 4. The data points favour the positive and crossing zero $\Delta G$ solutions.
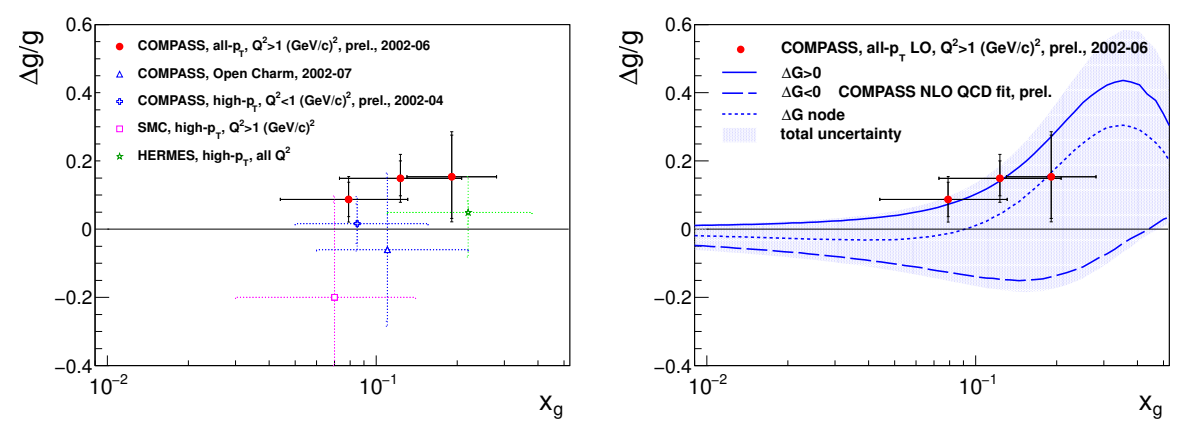

Figure 5. $\Delta g / g$ COMPASS results compared to other direct LO extractions (left) and COMPASS NLO QCD fits (right) at $3(\mathrm{GeV} / \mathrm{c})^{2}$.

\section{Measurements in the photoproduction regime via $A_{L L}\left(p_{T}\right)$}

Preliminary results of the double spin asymmetries of cross-sections for single hadron photoproduction are shown as a function of $p_{T}$ in Figs. 6 for a deuteron and a proton target [12]. These asymmetries found to be compatible with zero over the whole $p_{T}$ range except for high- $p_{T}$ in the deuteron case and low $p_{T}$ in the proton case. The gluon polarisation is probed by several subprocesses contributing to these measurements, like the PGF but also by subprocesses arising from the resolved photon contribution. Although theoretical calculation are available at NLO, it has been shown in [13, 14] on unpolarised data that for the low centre of mass energy available in COMPASS kinematics a resummation of soft gluons is mandatory to describe the data. The extension of the calculation for the polarised case is ongoing and do not permit a global QCD fit of the data yet.
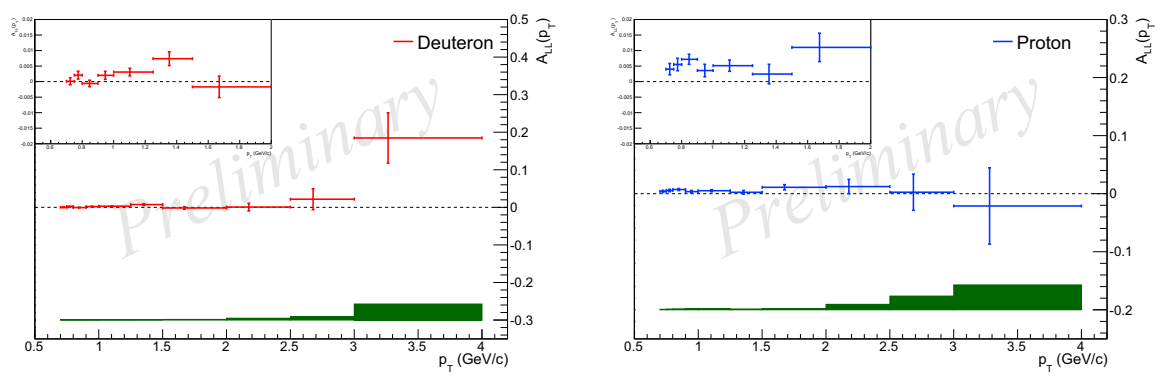

Figure 6. Double spin asymmetry $A_{L L}$ as a function of $p_{T}$ for the deuteron (left) and proton (right) target. The insets represent a zoom to the low $p_{T}$ region. 


\section{Quark fragmentation functions}

Although the quark fragmentation functions, $D_{q}^{h}$, do not constitute a result on the nucleon spin structure itself, these functions are of primary importance in the extraction of the quark helicity distributions per flavour in semi-inclusive deep inelastic scattering reactions. These structure functions are universal and described the process of hadronisation. They can be extracted via a QCD fit to the pion and kaon multiplicities, which represents the number of produced pions and kaons per deep inelastic events. Using the preliminary 500 data points of pion multiplicities measured on a deuteron target [15] and additional assumptions (including charged conjugation and isospin symmetry), a LO QCD fit is performed. Two quark fragmentation functions are fitted using the multiplicities of $\pi^{+}$and $\pi^{-}$: the "favoured" (when the quark of the nucleon is a valence quark of the produced hadron) and the "unfavoured" (otherwise). Preliminary results extracted at LO are shown in Fig. 7. They are in better agreement with the DSS ones than with the HKNS ones extracted from $e^{+} e^{-}$data exclusively.
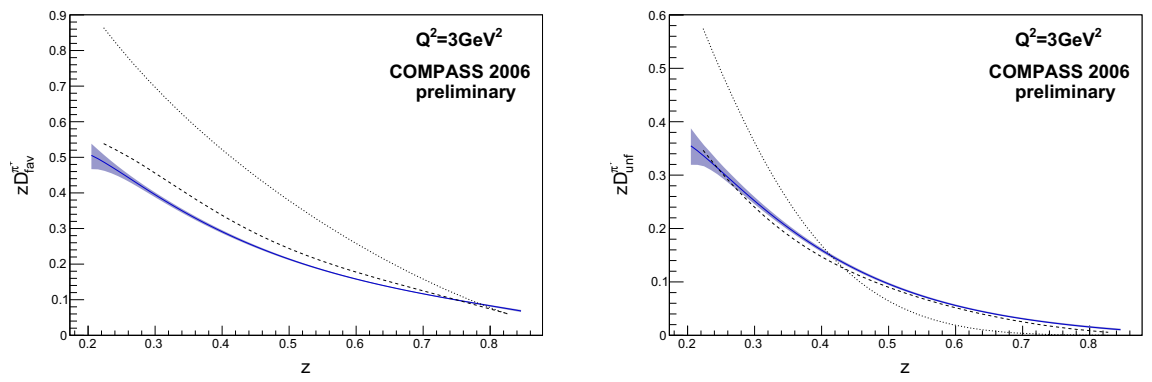

Figure 7. Favoured (left) and unfavoured (right) fragmentation functions as a function of the hadron energy fraction, $z$, for COMPASS (solid line), HKNS (dotted line) [16] and DSS (dashed line) [17] results.

\section{References}

[1] P. Abbon et al. (COMPASS Collaboration), NIMA 517, 455 (2007)

[2] V. Yu. Alexakhin et al. (COMPASS Collaboration), Phys. Lett. B 647, 8 (2007)

[3] V. Andrieux (COMPASS Collaboration), PoS DIS2013, 207 (2013)

[4] M. G. Alekseev et al. (COMPASS Collaboration), Phys. Lett. B 690, 466 (2010)

[5] J. Beringer et al. (Particle Data Group), Phys. Rev. D 86, 010001 (2012)

[6] M. Wilfert (COMPASS Collaboration), PoS DIS2014, 206 (2014)

[7] C. Adolph et al. (COMPASS Collaboration), Phys. Rev. D 87, 052018 (2013)

[8] B. Adeva et al. (Spin Muon Collabotation), Phys. Rev. D 58, 11002 (1998)

[9] M. Stolarski (COMPASS Collaboration), PoS DIS2014, 211 (2014)

[10] D. de Florian, R. Sassot, M. Stratmann and W. Vogelsang, Phys. Rev. Lett. 113, 012001 (2014)

[11] E. R. Nocera et al. (NNPDF Collaboration), arXiv:1406.5939v2[hep-ph]

[12] M. Levillain (COMPASS Collaboration), PoS DIS2014, 223 (2014)

[13] D. de Florian, M. Pfeuffer, A. Schafer and W. Vogelsang, Phys. Rev. D 88, 014024 (2013)

[14] C. Adolph et al. (COMPASS Collaboration), Phys. Rev. D 88, 091101 (2013)

[15] N. Du Fresne Von Hohenesche (COMPASS Collaboration), PoS DIS2014, 209 (2014)

[16] M. Hirai, S. Kumano, T.-H. Nagal and K. Sudoh, Phys. Rev. D 75, 094009 (2007)

[17] D. de Florian, R. Sassot and M. Stratmann, Phys. Rev. D 75, 114010 (2007) 